\title{
Homogeneous and Heterogeneous Combustion in Hydrogen-Fueled Catalytic Microreactors
}

\author{
Junjie Chen * and Baofang Liu
}

\author{
School of Mechanical and Power Engineering, Henan Polytechnic University, Jiaozuo, Henan, China \\ comcjj@yeah.net, baofang@escience.cn
}

\begin{abstract}
Keywords: Catalytic microreactor; Homogeneous combustion; Heterogeneous combustion; Flame stability; Hetero-/homogeneous interaction; Computational fluid dynamics.
\end{abstract}

\begin{abstract}
The hetero-/homogeneous combustion and interaction of hydrogen-fueled catalytic microreactors were investigated numerically. A two-dimensional CFD (computational fluid dynamics) model was developed, using elementary homogeneous and heterogeneous chemical reaction schemes, surface radiation heat transfer, heat conduction in the solid wall, and external heat losses. Computations were carried out to study the effects of the wall thermal conductivity, equivalence ratio, microreactor dimension, and inlet velocity on combustion characteristics, flame stability, and hetero-/homogeneous interaction. Despite the micro-scale, large transverse gradients in species mass fractions and temperature exist in the fluid and large axial gradients in temperature may exist in the walls. Wall thermal conductivity is crucial in determining the flame stability, as the walls transfer heat upstream for ignition of the cold incoming reactants but at the same time are responsible for heat losses. Combustible mixtures with compositions away from the stoichiometric point decrease the homogeneous chemistry contribution and the operating temperature. The microreactor dimension and inlet velocity have a strong effect on homogeneous flame stability. Smaller microreactors result in extinction because of the inhibition of homogeneous combustion induced by heterogeneous reaction; larger microreactors result in blowout due to the reduction of the heterogeneous contribution. Hetero-/homogeneous interaction maps were constructed in terms of microreactor dimension and inlet velocity.
\end{abstract}

\section{Introduction}

Hydrogen and hydrogen-containing fuels are under intense investigation for small- and large-scale power generation [1]. Hydrocarbons have an energy density significantly higher than that of the traditional lithium batteries that are currently used in cellular phones, laptops, and other portable electronics devices [2]. In the last years, microreactors for small portable power generation devices fueled with hydrogen or hydrogen-enriched hydrocarbons and syngas mixtures have been studied numerically and experimentally [3]. In such micro-scale systems hydrogen can be produced on-board from methane or high hydrocarbons using suitable microreformers [4]. Most microreactors operate with heterogeneous combustion or with a variety of hybrid concepts, namely combined hetero-/homogeneous combustion, rather than with pure homogeneous combustion. This is mainly dictated by the large surface-to-volume ratios of microreactors that result in much wider flame stability envelopes compared to those of pure homogeneous case, the existence of a multitude of undesirable flame instabilities in tight geometrical confinements and the efficient suppression of such instabilities by coating the microreactor walls with catalysts [5].

Although hybrid hetero-/homogeneous reactor designs have distinct homogeneous and heterogeneous combustion regions with the latter preceding the former, homogeneous combustion cannot always be ignored in the designated catalytic combustion region. Even at the large geometrical confinements of practical catalytic microreactors, homogeneous combustion can be appreciable for hydrocarbon fuels, depending on the residence time, temperature and pressure [6]. A detailed parametric study of fuel-lean hydrogen-air combustion in platinum-coated channels has delineated the regimes of channel hydraulic diameters, inlet temperatures, wall temperatures, and pressures for 
which homogeneous combustion amounts to at least $5 \%$ of the combined hetero-/homogeneous conversion [7]. Catalytic combustion of fuel-lean hydrogen-air mixtures is particularly challenging because of the diffusional imbalance of the deficient hydrogen reactant, resulting in superadiabatic surface temperatures that endanger the catalyst and reactor integrity. An inverse hybrid concept for hydrogen has been recently proposed to mitigate such superadiabatic effects, whereby the homogeneous combustion region precedes the heterogeneous combustion region [8].

In the last years, hetero-/homogeneous combustion of hydrogen at fuel-lean stoichiometries has been extensively studied. Appel et al. [9] numerically and experimentally investigated the homogeneous ignition of fuel-lean hydrogen-air mixtures over platinum in laminar channel-flow configurations, providing validated hetero-/homogeneous reaction schemes for fuel-lean hydrogen-air mixtures and addressing the underlying chemistry coupling. Maestri et al. [10] numerically and experimentally investigated the heterogeneous and homogeneous reactions of fuel-rich hydrogen-air mixtures over Rh-based catalysts at atmospheric pressure in nearly isothermal annular microreactors. The results shown that the low-temperature promotion of homogeneous conversion can be explained by the proper temperature-dependent enrichment of radical species. Zheng et al. [11] experimentally and numerically investigated the homogeneous ignition of hydrogen-air mixtures over platinum in optically accessible channel-flow reactors. Mantzaras et al. [12] extended the previous fuel-lean works for pressures up to 15 bar and preheats up to $790 \mathrm{~K}$, establishing regimes wherein the competition between the homogeneous and heterogeneous reaction pathways in conjunction with the intrinsic hydrogen homogeneous kinetics inhabited homogeneous combustion. Nonetheless, fundamental hetero-/homogeneous combustion studies of hydrogen-air mixtures over platinum at fuel-lean stoichiometries are still lacking in the literature.

The present work undertakes numerical investigations of hetero-/homogeneous combustion in hydrogen-fueled catalytic microreactors. Simulations were performed with a two-dimensional CFD model for the solid- and gas-phases that included elementary homogeneous and heterogeneous chemical reaction schemes, surface radiation heat transfer, heat conduction in the solid wall, and external heat losses. The objective of present work is to perform a comprehensive parametric study to understand the role of operating conditions, specifically, the wall thermal conductivity, equivalence ratio, microreactor dimension, and inlet velocity in determining flame stability and hetero-/homogeneous interaction.

\section{Numerical models and simulation approach}

\section{Geometric model}

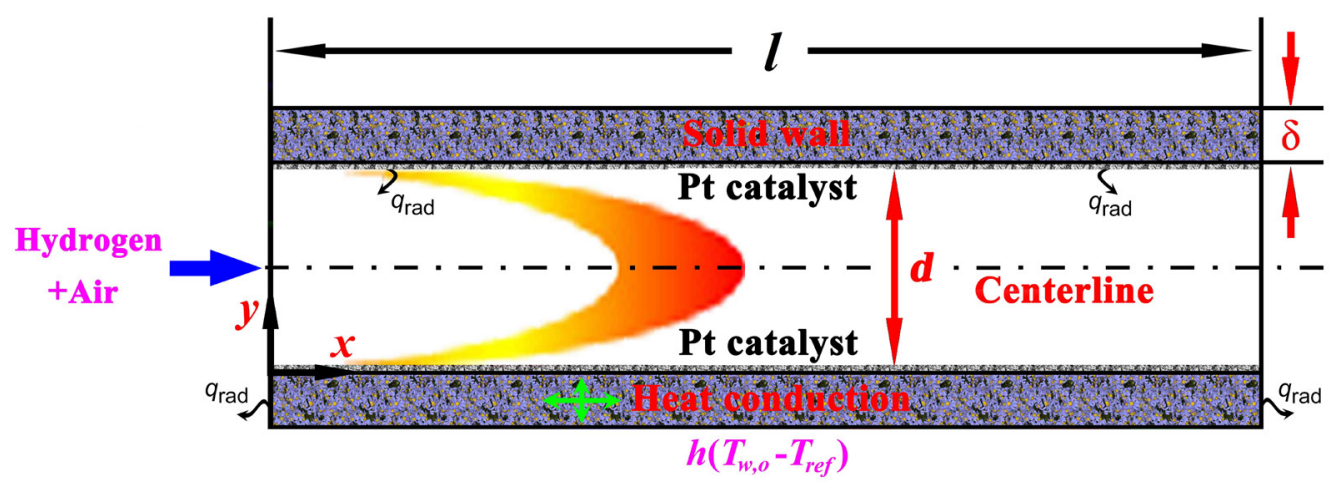

Fig. 1. Schematic diagram of the catalytic microreactor.

The channel-flow catalytic microreactor, illustrated in Fig. 1, consists of two parallel plates of length $l$, thickness $\delta$, and separation distance $d$ coated with platinum catalyst. Unless otherwise stated, the wall thickness is $0.2 \mathrm{~mm}$; the plates are separated by a gap size (height) of $0.8 \mathrm{~mm}$; inlet temperature and velocity are $300 \mathrm{~K}$ and $2 \mathrm{~m} / \mathrm{s}$, respectively; premixed hydrogen-air mixtures of equivalence ratio $\varphi$ is 0.8 . The parallel plate geometry implies that the third dimension (width) of the 
catalytic microreactor is much larger than the gap size. A two-dimensional CFD steady-state model was developed to simulate the coupling of the fluid flow and the combustion processes. Parametric studies were carried out by varying the wall thermal conductivity, equivalence ratio, and inlet velocity. Uniform profiles for the axial velocity, the temperature and the species mass fractions were used at the inlet.

\section{Mathematical model}

The governing equations for a steady reacting flow are shown below:

Continuity equation:

$$
\frac{\partial\left(\rho v_{x}\right)}{\partial x}+\frac{\partial\left(\rho v_{y}\right)}{\partial y}=0
$$

Momentum equation:

$$
\begin{aligned}
& \frac{\partial\left(\rho v_{x} v_{x}\right)}{\partial x}+\frac{\partial\left(\rho v_{x} v_{y}\right)}{\partial y}=-\frac{\partial p}{\partial x}+\frac{\partial \tau_{x x}}{\partial x}+\frac{\partial \tau_{x y}}{\partial y} \\
& \frac{\partial\left(\rho v_{y} v_{x}\right)}{\partial x}+\frac{\partial\left(\rho v_{y} v_{y}\right)}{\partial y}=-\frac{\partial p}{\partial y}+\frac{\partial \tau_{y x}}{\partial x}+\frac{\partial \tau_{y y}}{\partial y}
\end{aligned}
$$

Energy equation:

$$
\frac{\partial\left(\rho v_{x} h\right)}{\partial x}+\frac{\partial\left(\rho v_{y} h\right)}{\partial y}=\frac{\partial\left(k_{f} \partial T\right)}{\partial x^{2}}+\frac{\partial\left(k_{f} \partial T\right)}{\partial y^{2}}+\sum_{i}\left[\frac{\partial}{\partial x}\left(h_{i} \rho D_{i, m} \frac{\partial Y_{i}}{\partial x}\right)+\frac{\partial}{\partial y}\left(h_{i} \rho D_{i, m} \frac{\partial Y_{i}}{\partial y}\right)\right]-\sum_{i} h_{i} \dot{\omega}_{i} W_{i}
$$

Species equation:

$$
\frac{\partial\left(\rho Y_{i} v_{x}\right)}{\partial x}+\frac{\partial\left(\rho Y_{i} v_{y}\right)}{\partial y}=-\left[\frac{\partial}{\partial x}\left(\rho D_{i, m} \frac{\partial Y_{i}}{\partial x}\right)+\frac{\partial}{\partial y}\left(\rho D_{i, m} \frac{\partial Y_{i}}{\partial y}\right)\right]+\dot{\omega}_{i} W_{i}
$$

Surface species coverage equations:

$$
\frac{\partial \Theta_{i}}{\partial t}=\sigma_{i} \frac{\dot{s}_{i}}{\Gamma} \quad\left(i=N_{g}+1, \ldots, N_{g}+N_{s}\right)
$$

The energy equation in the reactor wall is given as follow:

$$
\frac{\partial\left(k_{s} \partial T\right)}{\partial x^{2}}+\frac{\partial\left(k_{s} \partial T\right)}{\partial y^{2}}=0
$$

\section{Chemical kinetics}

The elementary homogeneous scheme of by Burke et al. [13], which is a revised version of the Li et al. [14] mechanism, provided the platform for homogeneous chemistry. The elementary heterogeneous scheme of Deutschmann et al. [15] is employed to describe the hydrogen oxidation over platinum. The above heterogeneous and homogeneous reaction mechanisms have reproduced heterogeneous conversions and measured homogeneous ignition distances in fuel-lean and fuel-rich hydrogen-air combustion over platinum over a broader temperature and pressure range [16], and are consequently used in the ensuing simulations. Gaseous and surface thermodynamic data are included in the provided schemes. Mixture-average diffusion, including thermal diffusion for the light species, provides the gas-phase transport model. Homogeneous and heterogeneous reaction rates are evaluated with CHEMKIN and Surface-CHEMKIN, respectively.

\section{Computation scheme}

The fluid thermal conductivity, specific heat, and viscosity were calculated from a mass fraction weighted average of species properties, and the piecewise temperature polynomials were used for the calculation of the species-specific heat capacities. The boundary conditions are as follows. A flat, fixed velocity profile was used at the inlet. This boundary condition fixes the convective component of the flux of species and energy, but the diffusive component depends on the gradient of the computed temperature or species fields. Symmetry boundary conditions were employed at the centerline between the two plates. A fixed pressure was specified at the exit and far-field conditions were imposed for the rest of the variables. The heat flux at the fluid-wall interface was computed using Fourier's law and continuity in temperature and heat flux links the solid and fluid phases. The 
discrete ordinates (DO) model was used to consider the effect of interior surface to surface radiation. At exterior surfaces, the total heat-loss includes both natural convection and thermal radiation. A segregated solution solver with an under-relaxation method was used to solve the conservation equations. The second-order upwind scheme was used to discretize the model; the "SIMPLE" algorithm was used to couple the pressure and velocity. The convergence of CFD simulations was judged based on the residuals of all governing equations. All computations were performed on a cluster with $2 \times$ Xeon E5-2670 CPUs at 2.6-GHz. Computations were very demanding and its time varied from several hours to a few days depending on the combustion mode and geometry.

\section{Results and discussion}

\section{Combustion characteristics}

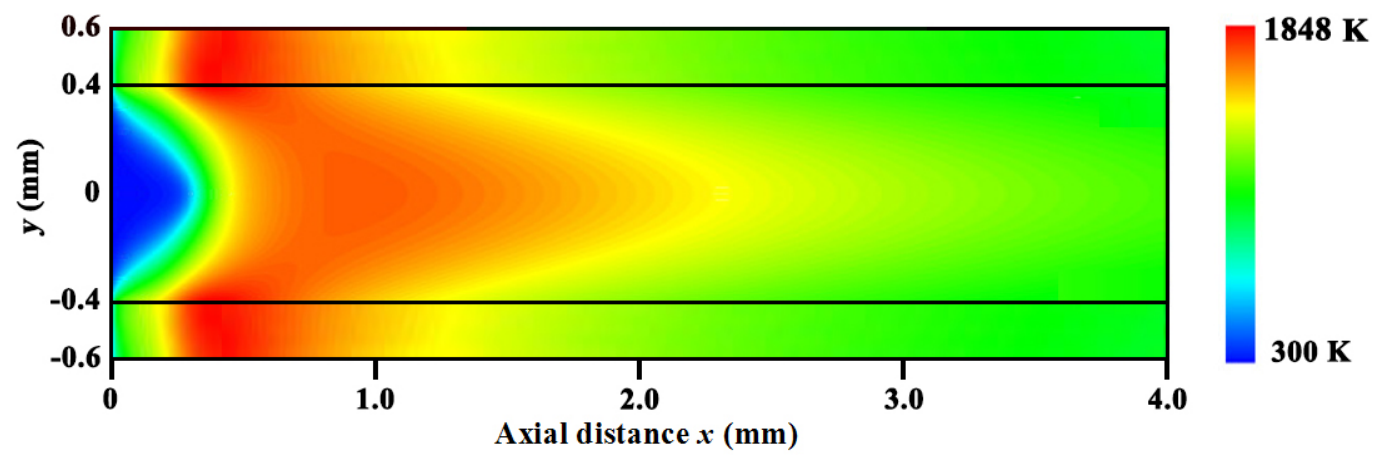

Fig. 2. The computed temperature contour for hetero-/homogeneous combustion of fuel-lean hydrogen-air mixtures.

Simulations were performed for a wide range of parameters. Fig. 2 illustrates CFD simulation results for a typical set of operating conditions and the nominal reactor geometry. The flame stabilizes in the center between the two plates. The reaction starts at the wall and travels towards the center as the flow goes downstream. Combustion occurs very rapidly, consuming most of the hydrogen in a very small region. Complete conversion is achieved, and a significant temperature rise is observed because of the exothermicity of the reaction. A microreactor typically exhibits three regions: the preheating, reaction, and post-reaction regions. While these regions are clearly demarcated in homogeneous microreactors, the preheating and reaction regions often overlap in catalytic microreactors. Despite the micro-scales, there are still significant transverse gradients in the reaction rate and fluid temperature until nearly the end of the reaction region. Despite the fluid transverse gradients, there are no significant transverse gradients within the walls themselves in all cases studied because of the short time-scale for conduction in the wall and their large aspect ratio.

\section{Effect of wall thermal conductivity}

Norton and Vlachos have demonstrated that the wall thermal conductivity plays an important role in the materials integrity and flame stability of microreactors [17, 18]. The wall plays a dual, competing role in the overall heat transfer. On one hand, it allows exterior heat losses, resulting in a delay ignition and consequently causing extinction. On the other hand, it provides a route for heat transfer from the post-combustion region to upstream for preheating that is necessary for ignition and flame stability. Note that the importance of wall thermal conduction on flame stability has been illustrated independently for Swiss roll type micro-combustors using an analytical treatment of the governing equations $[19,20]$.

The wall thermal conductivity affects the temperature profile within the wall and the possibility of hot spots. Fig. 3 shows the temperature profiles for the outer edge of the wall for different wall thermal conductivities. For low-wall-thermal-conductivity materials, significant axial temperature gradients are observed. Hotspot temperatures in excess of $1800 \mathrm{~K}$ can occur, an undesirable situation, as it exceeds the maximum operating temperatures of most materials of construction. Exceedingly 
high wall temperatures are characteristic of both micro- and macroscale thermally stabilized combustors [21, 22]. As the wall thermal conductivity increases, the wall temperature profiles become more uniform and the wall hot spot is eliminated. Despite the apparent advantages of a higher wall thermal conductivity for material stability, most materials that offer high conductance are metals, and therefore would not be inert to radical quenching. A more reasonable solution would be thicker walls of a more inert material that may have a lower thermal conductivity.

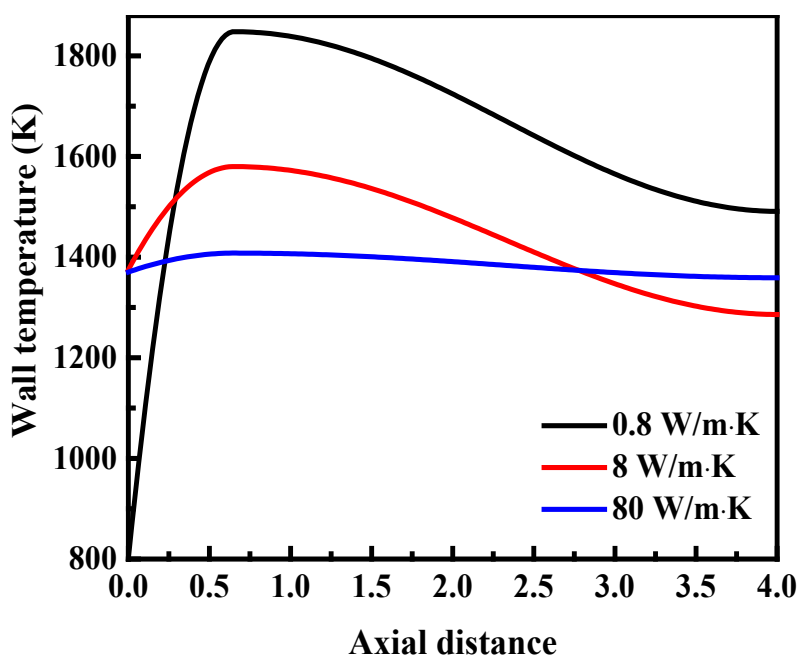

Fig. 3. Wall outer edge temperature profiles for different wall thermal conductivities. High wall thermal conductivity result in uniform temperature profiles without hotspots. Low wall thermal conductivities leads to large axial wall-temperature gradients and high maximum temperatures.

\section{Effect of equivalence ratio}

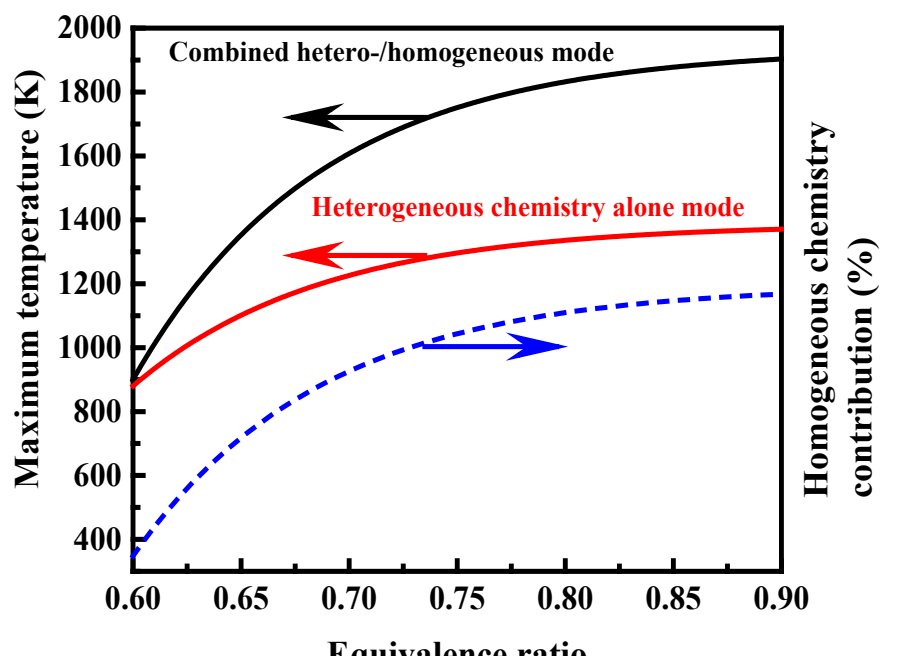

Fig. 4. Maximum temperature at the symmetry plane for heterogeneous chemistry alone and combined hetero-/homogeneous mode as well as homogeneous chemistry contribution for the combined hetero-/homogeneous mode as a function of equivalence ratio.

In order to investigate the effect of equivalence ratio, simulations were performed for various modes: homogeneous chemistry alone, heterogeneous chemistry alone, and combined hetero-/homogeneous chemistries. The pure heterogeneous chemistry solutions were obtained by turning off the homogeneous chemistry. Fig. 4 shows the maximum temperature at the symmetry plane as a function of the equivalence ratio for heterogeneous chemistry alone and combined hetero-/homogeneous mode, along with the homogeneous chemistry contribution as a function of the equivalence ratio for combined hetero-/homogeneous mode. The temperature decrease with 
decreasing equivalence ratio occurs not only due to the reduction in fuel composition but also due to a decrease in the homogeneous chemistry contribution. For sufficiently fuel-lean compositions, very little homogeneous chemistry actually occurs. Below a critical composition (approximately 0.6), extinction occurs. This equivalence ratio is in reasonable agreement with experimental data obtained under similar conditions $[23,24]$.

\section{Effect of microreactor dimension and inlet velocity}

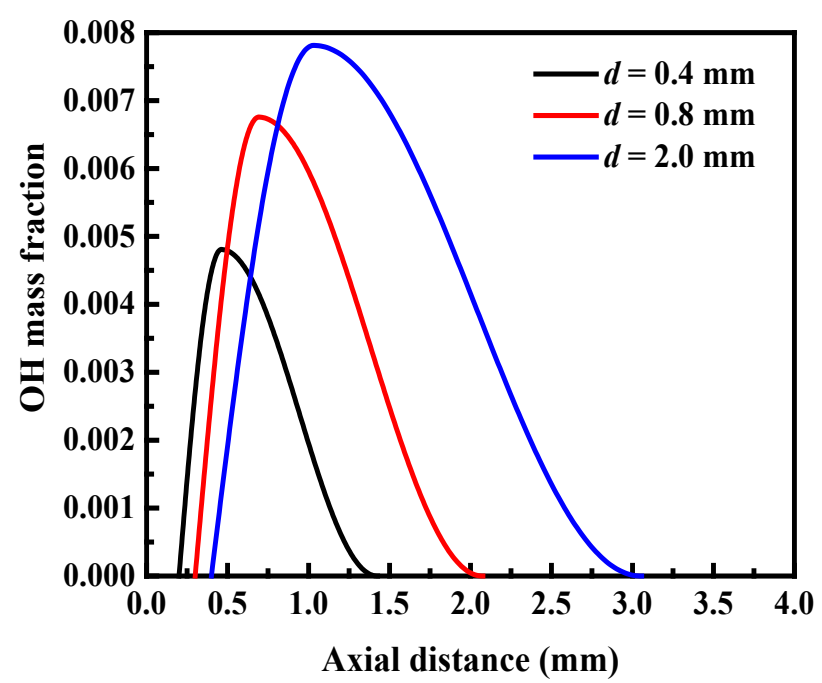

Fig. 5. OH concentration contour at the symmetry plane for different microreactor dimensions.

Here we investigate the combustion characteristics in hydrogen-fueled catalytic microreactors when varying the separation distance between the two plates. Fig. 5 shows the $\mathrm{OH}$ concentration contour at the symmetry plane for different microreactor dimensions. The $\mathrm{OH}$ concentration becomes weaker and shifts upstream with decreasing the microreactor dimension, as a result of the enhancement of heterogeneous reaction that is affected by the mass diffusion time. For smaller microreactors, the fuel in the bulk gas needs less diffusive time to reach the catalytic surface. The radicals and heat produced also help to maintain homogeneous combustion with higher inlet velocities.

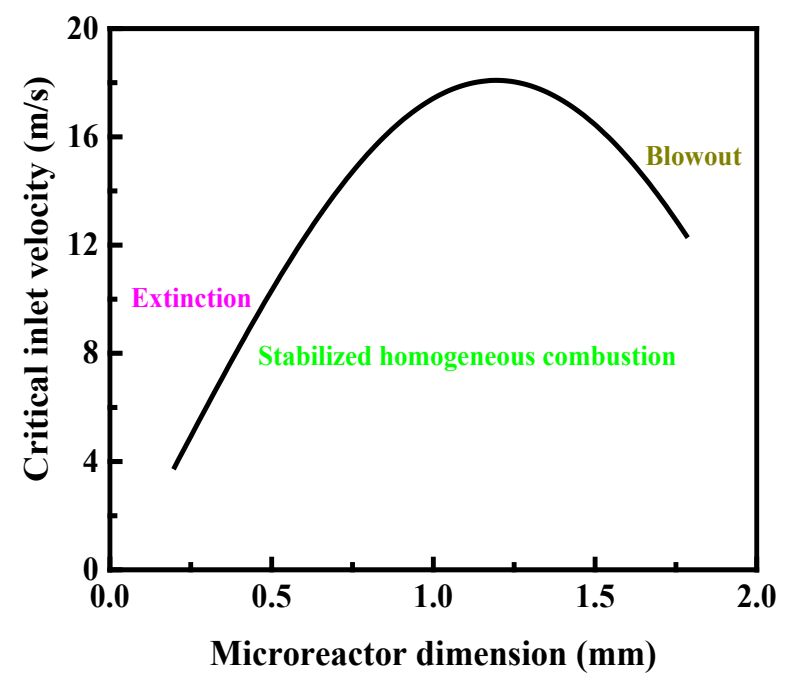

Fig. 6. Critical velocity as a function of microreactor dimension. The left curve represents the low-dimension limit, resulting in extinction. The right curve represents the high-dimension limit, resulting in blowout.

Parametric continuation is used to move from one stationary solution to another. When the solution reaches a turning point or homogeneous combustion cannot be sustained, this is denoted as a 
critical point. Knowledge of critical parameter values of the inlet velocity gives a better understanding of the important factors controlling flame stability. These critical values are useful as guides, but actual values will vary depending on the system of interest.

Fig. 6 shows the critical inlet velocity for homogeneous combustion in hydrogen-fueled catalytic microreactors with different microreactor dimensions. As the microreactor dimension is increased, the critical inlet velocity first increases and then decreases. The left curve represents the low-dimension limit, resulting in extinction because of the inhibition of homogeneous combustion induced by heterogeneous reaction. The right curve represents the high-dimension limit, resulting in blowout due to the reduction of the heterogeneous contribution. Between these curves stabilized homogeneous combustion is allowed, whereas outside the envelope, sustained homogeneous combustion is impossible.

\section{Hetero-/homogeneous interaction}

Earlier fundamental studies of hetero-/homogeneous systems focused on wires, foils, and large-scale systems, which are not easily related to operation at micro-scales [25, 26]. Recently, several investigations regarding hetero-/homogeneous reactions in catalytic microreactors were performed using computational fluid dynamics simulations [27, 28].

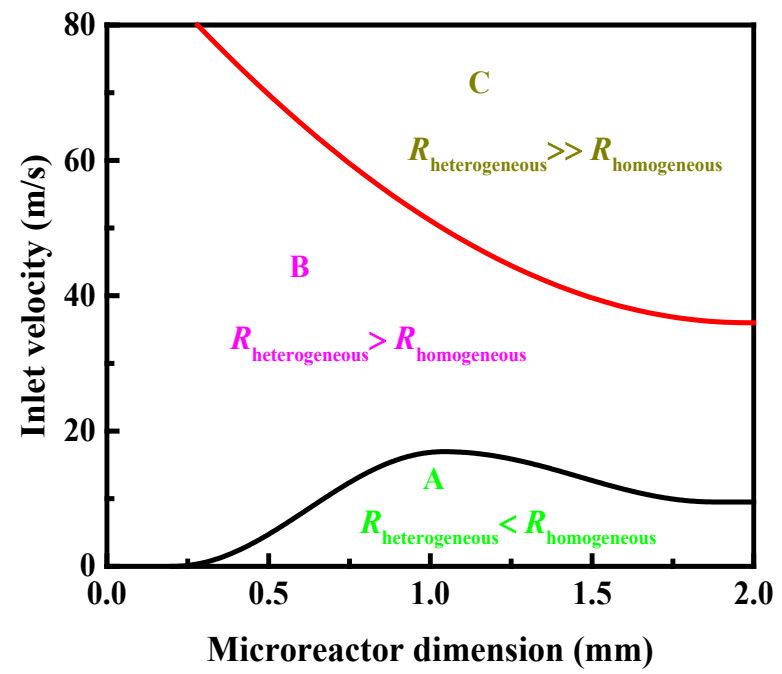

Fig. 7. Hetero-/homogeneous interaction in terms of microreactor dimension and inlet velocity.

In catalytic microreactors, the hetero-/homogeneous interaction can be divided into three types. A: the homogeneous reaction is inhibited by the presence of the heterogeneous reaction but still can be sustained over a large range of inlet velocity; B: the homogeneous reaction is obviously inhibited by heterogeneous reaction, and consequently have little effect on the flame stability and combustion characteristics; C: homogeneous reaction can be completely ignored. The catalytic microreactor is dominated by heterogeneous reaction. In conclusion, these hetero-/homogeneous interaction types can be schematically constructed in terms of microreactor dimension and inlet velocity, as shown in Fig. 7. In region A, as the microreactor dimension is relatively large and the inlet velocity is low, homogeneous reaction is affected by heterogeneous reaction but still be sustained in catalytic microreactors; the homogeneous reaction rate obviously exceeds the heterogeneous counterpart. As the microreactor dimension decreases or the inlet velocity increases, the characteristic reaction moves into region $\mathrm{B}$ where the heterogeneous reaction is enhanced and surpasses homogeneous reaction, and consequently dominates the combustion process. As operation conditions are in region $\mathrm{C}$, with relatively large microreactor dimension and very high inlet velocity, the homogeneous reaction is entirely inhibited to have almost no effects. 


\section{Conclusions}

The hetero-/homogeneous combustion processes hydrogen-fueled catalytic microreactors coated with platinum and fed with premixed fuel-lean hydrogen-air mixtures were investigated numerically. A two-dimensional CFD numerical model was employed that included detailed hetero-/homogeneous reaction schemes and heat transfer mechanisms to study the effects of the wall thermal conductivity, equivalence ratio, microreactor dimension, and inlet velocity on combustion characteristics, flame stability, and hetero-/homogeneous interaction. The following are the key conclusions of this study.

- The catalytic microreactor can be divided into a preheating, a reaction, and a post-reaction region. The preheating and the reaction regions often overlap.

- Despite the micro-scale of these systems, large transverse gradients in species mass fractions and temperature exist in the fluid and large axial gradients in temperature may exist in the walls.

- The wall thermal conductivity plays a competing role in flame stability. Walls transfer heat upstream for ignition of the cold incoming reactants but at the same time are responsible for heat losses.

- Regarding material lifetimes, higher wall thermal conductivities reduce the hotspots and wall temperature gradients, and should be preferred.

- Combustible mixtures with compositions away from the stoichiometric point decrease the homogeneous chemistry contribution and the operating temperature.

- The microreactor dimension and inlet velocity have a strong effect on homogeneous flame stability.

- Microreactor dimension plays a competing role in homogeneous flame stability. Smaller microreactors result in extinction because of the inhibition of homogeneous combustion induced by heterogeneous reaction. On the other hand, larger microreactors result in blowout due to the reduction of the heterogeneous contribution. As a result, there is only a relatively narrow envelope of inlet velocities within which homogeneous combustion can be stabilized.

- The hetero-/homogeneous interaction can be divided to three types. A: the homogeneous reaction is inhibited by the presence of the heterogeneous reaction but still can be sustained over a large range of inlet velocity; $\mathrm{B}$ : the homogeneous reaction is obviously inhibited by heterogeneous reaction, and consequently have little effect on the flame stability and combustion characteristics; $\mathrm{C}$ : homogeneous reaction can be completely ignored.

\section{Acknowledgements}

This work was supported by the National Natural Science Foundation of China (No. 51506048).

\section{References}

[1] R. Sui, N.I. Prasianakis, J. Mantzaras, N. Mallya, J. Theile, D. Lagrange, and M. Friess, An experimental and numerical investigation of the combustion and heat transfer characteristics of hydrogen-fueled catalytic microreactors, Chem. Eng. Sci. 141 (2016) 214-230.

[2] I. Alam, D.H. West, and V. Balakotaiah, Transport effects on pattern formation and maximum temperature in homogeneous-heterogeneous combustion, Chem. Eng. J. 288 (2016) 99-115.

[3] G.D. Stefanidis and D.G. Vlachos, Controlling homogeneous chemistry in homogeneous-heterogeneous reactors: Application to propane combustion, Ind. Eng. Chem. Res. 48 (2009) 5962-5968.

[4] L. Huang, J. Xie, W. Chu, R. Chen, D. Chu, and A.T. Hsu, Iron-promoted nickel-based catalysts for hydrogen generation via auto-thermal reforming of ethanol, Catal. Commun. 10 (2009) 502-508. 
[5] A. Brambilla, C.E. Frouzakis, J. Mantzaras, A. Tomboulides, S. Kerkemeier, and K. Boulouchos, Detailed transient numerical simulation of $\mathrm{H}_{2} /$ air hetero-/homogeneous combustion in platinum-coated channels with conjugate heat transfer, Combust. Flame 161 (2014) 2692-2707.

[6] S. Karagiannidis, J. Mantzaras, and K. Boulouchos, Stability of hetero-/homogeneous combustion in propane- and methane-fueled catalytic microreactors: Channel confinement and molecular transport effects, Proc. Combust. Inst. 33 (2011) 3241-3249.

[7] M. Baigmohammadi, S.Tabejamaat, and J. Zarvandi, Numerical study of the behavior of methane-hydrogen/air pre-mixed flame in a micro reactor equipped with catalytic segmented bluff body, Energy 85 (2015) 117-144.

[8] Y. Ghermay, J. Mantzaras, R. Bombach, and K. Boulouchos, Homogeneous combustion of fuel-lean $\mathrm{H}_{2} / \mathrm{O}_{2} / \mathrm{N}_{2}$ mixtures over platinum at elevated pressures and preheats, Combust. Flame 158 (2011) 1491-1506.

[9] C. Appel, J. Mantzaras, R. Schaeren, R. Bombach, A. Inauen, B. Kaeppeli, B. Hemmerling, and A. Stampanoni, An experimental and numerical investigation of homogeneous ignition in catalytically stabilized combustion of hydrogen/air mixtures over platinum, Combust. Flame 128 (2002) 340-368.

[10]M. Maestri, A. Beretta, T. Faravelli, G. Groppi, and E. Tronconi, Role of gas-phase chemistry in the rich combustion of $\mathrm{H}_{2}$ and $\mathrm{CO}$ over a $\mathrm{Rh} / \mathrm{Al}_{2} \mathrm{O}_{3}$ catalyst in annular reactor, Chem. Eng. Sci. 62 (2007) 4992-4997.

[11]X. Zheng, J. Mantzaras, and R. Bombach, Kinetic interactions between hydrogen and carbon monoxide oxidation over platinum, Combust. Flame 161 (2014) 332-346.

[12]J. Mantzaras, R. Bombach, and R. Schaeren, Hetero-/homogeneous combustion of hydrogen/air mixtures over platinum at pressures up to 10 bar, Proc. Combust. Inst. 32 (2009) 1937-1945.

[13]M.P. Burke, M. Chaos, Y. Ju, F.L. Dryer, and S.J. Klippenstein, Comprehensive $\mathrm{H}_{2} / \mathrm{O}_{2}$ kinetic model for high-pressure combustion, Int. J. Chem. Kinet. 44 (2012) 444-474.

[14]J. Li, Z. Zhao, A. Kazakov, and F.L. Dryer, An updated comprehensive kinetic model of hydrogen combustion, Int. J. Chem. Kinet. 36 (2004) 566-575.

[15]O. Deutschmann, L.I. Maier, U. Riedel, A.H. Stroemman, and R.W. Dibble, Hydrogen assisted catalytic combustion of methane on platinum, Catal. Today 59 (2000) 141-150.

[16]H.A. Uranakara, S. Chaudhuri, H.L. Dave, P.G. Arias, and H.G. Im, A flame particle tracking analysis of turbulence-chemistry interaction in hydrogen-air premixed flames, Combust. Flame 163 (2016) 220-240.

[17]D.G. Norton and D.G. Vlachos, A CFD study of propane/air microflame stability, Combust. Flame 138 (2004) 97-107.

[18]D.G. Norton and D.G. Vlachos, Combustion characteristics and flame stability at the microscale: a CFD study of premixed methane/air mixtures, Chem. Eng. Sci. 58 (2003) 4871-4882.

[19]C.H. Kuo and P.D. Ronney, Numerical modeling of non-adiabatic heat-recirculating combustors, Proc. Combust. Inst. 31 (2007) 3277-3284.

[20]P.D. Ronney, Analysis of non-adiabatic heat-recirculating combustors, Combust. Flame 135 (2003) 421-439.

[21]A.D. Stazio, C. Chauveau, G. Dayma, and P. Dagaut, Combustion in micro-channels with a controlled temperature gradient, Exp. Therm Fluid Sci. 73 (2016) 79-86. 
[22]E. Miyata, N. Fukushima, Y. Naka, M. Shimura, M. Tanahashi, and T. Miyauchi, Direct numerical simulation of micro combustion in a narrow circular channel with a detailed kinetic mechanism, Proc. Combust. Inst. 35 (2015) 3421-3427.

[23]C.-H. Leu, S.-C. King, J.-M. Huang, C.-C. Chen, S.-S. Tzeng, C.-I. Lee, W.-C. Chang, C.-C. Yang, Visible images of the catalytic combustion of methanol in a micro-channel reactor, Chem. Eng. J. 226 (2013) 201-208.

[24]D.G. Norton and D.G. Vlachos, Hydrogen assisted self-ignition of propane/air mixtures in catalytic microburners, Proc. Combust. Inst. 30 (2005) 2473-2480.

[25]E.D. Tolmachoff, A.D. Booth, I.C. Lee, W.R. Allmon, and C.M. Waits, Modeling and experimental analysis of $n$-dodecane oxidation in platinum-coated channels, Combust. Flame 162 (2015) 3674-3680.

[26]K. Gosiewski, Y.S. Matros, K. Warmuzinski, M. Jaschik, and M. Tanczyk, Homogeneous vs. catalytic combustion of lean methane-air mixtures in reverse-flow reactors, Chem. Eng. Sci. 63 (2008) 5010-5019.

[27]S.W. Jeon, W.J. Yoon, M.W. Jeong, and Y. Kim, Optimization of a counter-flow microchannel reactor using hydrogen assisted catalytic combustion for steam reforming of methane, Int. J. Hydrogen Energy 39 (2014) 6470-6478.

[28]G.A. Boyarko, C.-J. Sung, and S.J. Schneider, Catalyzed combustion of hydrogen-oxygen in platinum tubes for micro-propulsion applications, Proc. Combust. Inst. 30 (2005) 2481-2488. 